\title{
A atuação do cirurgião dentista na odontogeriatria: uma revisão de literatura
}

\author{
The dentist surgeon's performance in odontogeriatry: a literature review
}

El desempeño del cirujano dental en odontogeriatría: una revisión de la literatura

José Milton de Aquino e Silva Neto ${ }^{1 *}$, Daysielle Rodrigues de Oliveira', Karollyne Rodrigues Pereira1, João Vitor Brasil Alves Rodrigues Almeida Barros', Cibelle Teresa de Albuquerque Machado", Michelle Leão Bittencourt Brandão Medeiros ${ }^{1}$, Tayguara Cerqueira Cavalcanti .

\section{RESUMO}

Objetivo: Analisar o papel do Cirurgião Dentista (CD) no atendimento aos pacientes idosos, buscando expor as especificidades da assistência geriátrica, como também apresentar ocorrências comuns ao paciente idoso que venham ser um fator limitante ao seu tratamento. Métodos: procedeu-se através de uma revisão de literatura, com interpelação descritiva e informativa, em que a metodologia de preceitos se deu por meio de buscas por literaturas cientificas, em bases de dados, tendo como descritores: Odontologia geriátrica, os cuidados da cavidade oral em pacientes idosos e atendimentos especializados. Resultados: O CD no âmbito geriátrico executa um emprego no aspecto da integralidade multiprofissional, visando a promoção da saúde bucal em pacientes da terceira idade, levando em consideração sempre as condições sistêmicas e psicossociais, além de analisar as inúmeras alterações ocorridas pelo processo natural de envelhecimento. Considerações Finais: No Brasil a crescente população de pessoas idosas tem aumentado de forma vertiginosa. $O C D$ deve situar-se a essas circunstâncias e amplificar suas compreensões na área da odontogeriatria para só assim assegurar um recurso terapêutico preciso, buscando sempre o conforto desses pacientes, já que eles necessitam de uma atenção mais especifica buscando precisão nos prognósticos e diagnósticos.

Palavras-chave: Assistência integral à saúde, Assistência ao paciente, Higiene bucal, Idoso.

\begin{abstract}
Objective: Analyze the role of the dental surgeon $(C D)$ in the care of elderly patients, seeking to expose the specificities of geriatric care, as well as to present common occurrences to elderly patients that may be a limiting factor in their treatment. Methods: Proceeded through a literature review, with descriptive and informative interpellation, in which the methodology of precepts took place through searches for scientific literature, in databases, having as descriptors: Geriatric dentistry, oral cavity care in elderly patients and specialized care. Results: The CD in the geriatric scope performs a job in the aspect of multiprofessional integrality, aiming at the promotion of oral health in elderly patients, always taking into account the systemic and psychosocial conditions, in addition to analyzing the countless changes occurred by the natural aging process. Final Considerations: In Brazil, the growing population of elderly people has increased dramatically, the CD must situate itself in these circumstances and amplify its understandings in the area of odontogeriatrics in order to ensure an accurate therapeutic resource, always seeking the comfort of these patients, since they need more specific attention seeking precision in the prognoses and diagnoses.
\end{abstract}

Key Words: Comprehensive health care, Patient care, Oral hygiene, Aged.

\footnotetext{
${ }^{1}$ Centro de Ensino Superior de Maceió (CESMAC), Maceió - Alagoas.

*E-mail: milton_neto_166@hotmail.com
} 


\section{RESUMEN}

Objetivo: Analice el papel del cirujano dental (CD) en la atención de pacientes de edad avanzada, buscando exponer las especificidades de la atención geriátrica, así como presentar casos comunes a pacientes de edad avanzada que pueden ser un factor limitante en su tratamiento. Métodos: Procedió a través de una revisión de la literatura, con interpelación descriptiva e informativa en la cual la metodología de los preceptos se llevó a cabo a través de búsquedas de literatura científica, en bases de datos, teniendo como descriptores: odontología geriátrica, cuidado de la cavidad oral en pacientes de edad avanzada y atención especializada. Resultados: EI CD en el ámbito geriátrico realiza un trabajo en el aspecto de la integralidad multiprofesional, con el objetivo de promover la salud bucal en pacientes de edad avanzada, siempre teniendo en cuenta las condiciones sistémicas y psicosociales, además de analizar los innumerables cambios producidos por el proceso de envejecimiento natural. Consideraciones finales: En Brazil, la creciente población de personas mayores ha aumentado dramaticamente. EI CD debe situarse en estas circunstancias y ampliar sus conocimientos en el área de odontogeriatría para garantizar un recurso terapéutico preciso, siempre buscando la comodidad de estos pacientes, ya que necesitan atención más específica que busque precisíon en los pronósticos y diagnósticos.

Palabras Clave: Atención integral de salud, Atención al paciente, Higiene bucal, Anciano.

\section{INTRODUÇÃO}

O grande avanço da população com uma faixa etária de idade maior que 59 anos é um acontecimento ocorrido a nível mundial, do qual vem expressando-se significativamente em países emergentes (MORAES E, 2012). Estima-se que até o ano de 2025 o Brasil ocupará a sexta posição mundial no que se refere a esse grupo populacional. Concede este fato do envelhecimento da população ser tão expressiva devido ao fato da diminuição de taxa de fecundidade esta correlacionada à diminuição de mortalidade infantil e a ampliação da probabilidade ao que se refere a expectativa de vida (BARBOSA LNF, et al., 2015).

A portaria no 2.528 , de 22 de fevereiro de 2006, faz uma atualização ao que se refere as Políticas Nacionais de Saúde da Pessoa Idosa (geriátrica), sugerindo como orientação de assistência do "Envelhecimento Ativo", buscando a otimização e qualidade ao que se refere a saúde, colaboração e segurança, com o intuito de aprimorar e melhorar o estilo de vida à medida que os cidadãos vão ficando com maior idade (MENDES E, 2011).

Dentro do entendimento sobre o envelhecimento ativo e saudável, salienta-se a odontogeriatria, visto que corresponde-se a uma especialidade odontológica dos quais os Cirurgiões Dentistas buscam estabelecer um tratamento mais qualificado, cuidadoso e acolhedor, do qual tem como foco crucial os cuidados com os cidadãos de determinada população, focando-se especialmente nos cuidados terapêuticos de forma preventiva e curativa dos pacientes que possuem predisposição no âmbito sistêmico ou crônico dos quais são relacionadas a doenças de aspectos fisiológicos ou patológicos (BERKEY DB e SCANNAPIECO FA, 2013).

Esses princípios modernos estabelecidos na área odontológica é estabelecido através do enquadramento desde o início da grade curricular da graduação, até sua formação, onde busca fazer uma boa anamnese no primeiro momento sem focar apenas nas queixas principais que os levaram ao consultório odontológico, desse jeito, as conjunções sistemáticas e especificas de cada paciente são observadas, levando assim em consideração o que pode interferir no plano de tratamento e no diagnóstico final (COSTA DR, et al., 2015).

Os princípios referentes à atenção primaria em particular referente aos idosos surgiu como forma de estabelecimento pautado na fortificação das organizações dos vínculos agrupados pela saúde do indivíduo de uma forma particularizada como comunitária, de conformação delimitada em áreas de abrangências, continua e estruturadas, pelo meio de medidas preventiva e interventiva (BERKEY DB e SCANNAPIECO FA, 2013).

As cautelas e prevenções impostas aos pacientes que possuem uma maior idade, dos quais estão em um determinado grupo de maior risco de doenças sistêmicas dos quais os aspectos históricos médicos deparamse com possíveis estabelecimentos de fatores limitantes no que se refere aos aspectos orgânicos de modo 
frequente relacionada ao processo de envelhecimento natural, deve ser estabelecida uma assistência mais humanitária e atenciosa das equipes multiprofissionais (SOWMYA MK, et al., 2011).

Levando em consideração os enfoques no que se refere à saúde bucal dos quais é um constituinte de relevância mundial, tendo que se destacar os princípios de suas inclusões nas obrigações de qualificações e analises da saúde integral levando em consideração está uma potencialidade nos atributos de peculiaridade de vida por completo (BIZERRIL DO, et al., 2016).

O dano ou até mesmo a perda devido extrações de algum elemento dental são estabelecidas e agregadas com uma forma de repercussões e choques na autoestima dos pacientes nos aspectos referentes à funcionalidade, estéticos, fenômenos emocionais e inter-relação no âmbito social, além disto, é classificado como uma seriedade presente na saúde pública, visto que afeta de forma direta a autoestima dos pacientes (AGOSTINHO ACMG, et al., 2015).

Tendo como consideração a pouca existência de campanhas e programas odontológicos direcionados para as pessoas de terceira idade, a Odontologia carece estabelecer práticas integradoras referentes a essas pessoas, seja ela de forma preventiva ou até mesmo de estabelecimento curativo, dos quais possuam uma ideologia e interpretação acurada a respeito deixa faixa etária, e no tocante processo de envelhecimento de uma maneira geral (JANSSON MM, et al.,2017).

É de suma importância a relevância do conhecimento nos aspectos referentes às modificações ocasionadas por processos fisiológicos e patológicos que prejudicam de forma direta o organismo dos pacientes geriátricos, além dos enfoques das relações sociais referentes a psicologia do indivíduo presente.

O Cirurgião Dentista (CD) deve possuir uma relação direta com o médico geriatra responsável pelo paciente, com o objetivo de dialogar sobre os aspectos relacionados à saúde de maneira geral, os medicamentos que estão sendo administrados, tendo como consideração que a grande maioria dos idosos fazem uso de determinadas drogas e muito das vezes pode acarretar efeitos colaterais.

A função do Cirurgião Dentista é obter uma conexão diretamente com esses indivíduos tendo como objetivo fundamental o não comprometimento da saúde bucal (BIZERRIL DO, et al., 2016).

Desta maneira, o Cirurgião Dentista que possuem uma capacitação e habilidade possuem como fundamental desígnio averiguar e estabelecer exames clínicos oportunos em idosos que precisem de determinados cuidados buscando sempre acompanhar de forma direta, visando diminuir possíveis modificações da microbiota do meio bucal, se preciso for é necessário sempre remover focos bacterianos que sejam capazes de acarretar eventuais infecções, prejudicando assim o bem-estar do paciente idoso.

O CD pode fazer desde uma simples restauração em algum elemento dental até mesmo restabelecer através de cirurgia a saúde do idoso. Deste modo, é expresso a real importância de que os recursos terapêuticos médicos estejam sempre administrados de forma correta, tendo em conhecimento dessas administrações os profissionais da área odontológica (AMES NJ, et al., 2011). A benfeitoria prestada aos pacientes idosos referentes à saúde oral realizadas pelos Dentistas reduzem de forma elevada o aparecimento de adoecimentos pertencentes ao meio bucal, como também ao sistema respiratório e a multiplicação de contágios em outros sistemas presentes nos órgãos (RABELO GD, et al., 2010).

A forma integrativa de trabalhar com uma equipe multidisciplinar na atenção básica de saúde propõe-se, acima de tudo, às precauções que possam a vim ser riscos à saúde do idoso, além disso, têm que ser levado em consideração em que esse grupo se encontra, nos aspectos emotivos, nível de instrução, a falta de locomoção, moradias longes de alguns centros de atenções à saúde (BIZERRIL DO, et al., 2016).

Compreendendo-se a importância que a Odontogeriatria possui no comprometimento do bem estar do idoso e percebendo a elevada relevância das equipes multiprofissionais, o presente trabalho tem como prisma examinar pelo meio de uma revisão de literatura o real valor e funcionamento do papel dos Cirurgiões Dentistas no contexto da priorização aos idosos, desde a sua introdução de uma simples consulta corriqueira, até as ações prestadas em ocorrências nos eixos de possíveis transtornos das infecções bucais, dos quais são aptos de acometer ainda por cima a saúde desses pacientes. 


\section{MÉTODOS}

Este trabalho foi estabelecido por meio de uma revisão de literatura estabelecida entre os períodos de dezembro de 2019 a março de 2020, onde foram avaliados artigos publicados em bases de dados eletrônicos LILACS (Literatura Latino Americana e do Caribe em Ciências da Saúde), MEDLINE (Literatura Internacional em Ciência da Saúde) e SCIELO (Scientific Electronic Library Online) e livros que estavam disponíveis nos acervos bibliográficos presentes na biblioteca central do Centro Universitário CESMAC (Centro de Ensino Superior de Maceió).

Para tal fim a revisão de literatura foi determinada por meio das seguintes diretrizes: Os desenhos dos estudos deveriam ser ensaios clínicos controlados randomizados em humanos, na linguagem, portuguesa e inglesa, estabelecidos os trabalhos publicados nos últimos 8 anos (2012 a 2020) que se mencionaram aos procedimentos estabelecidos no sistema único de saúde a respeito dos cuidados com os idosos, as abrangências humanizadas do atendimento odontogeriatrico, possuindo como processo de exclusão os períodos de publicações, relatos de casos, ensaios não controlados, monografias e os artigos que não possuíam relevância com a temática, sendo eleito os textos científicos que apresentavam na íntegra o real objetivo do trabalho, levando em consideração os atendimentos geriátricos na odontologia, buscando dessa forma a melhor técnica e conscientização para a obtenção de resultados satisfatórios e benéficos para a correlação entre profissional e paciente. Os descritores utilizados foram: Assistência Integral à Saúde, Assistência ao Paciente, Higiene Bucal, Idoso.

\section{RESULTADOS E DISCUSSÃO}

Inicialmente, foram obtidos 24 artigos ao que se referia a atenção estabelecida ao paciente idoso na odontogeriatria. Dos quais, 10 estavam repetidos nas referidas bases de dados, ficando 14 artigos para o processo de leitura dos títulos, os quais 4 foram excluídos por não corresponder as principais adaptações dos critérios que estavam sendo focados. 10 artigos designados para observação dos resumos, dos quais três foram eliminados, ficando apenas 7 para assegurar esse estudo. Após a revisão das referências dos artigos nenhum outro trabalho foi incorporado.

Ao fim das investigações dos dados, a revisão foi mesclada ocorrendo o processo de miscigenação dos sete artigos, e o fluxograma da Figura 1 mostra de forma clara todos os artifícios de busca pelas pesquisas elegidas para a confecção desta revisão. 
Figura 1 - Fluxograma dos estudos identificados.

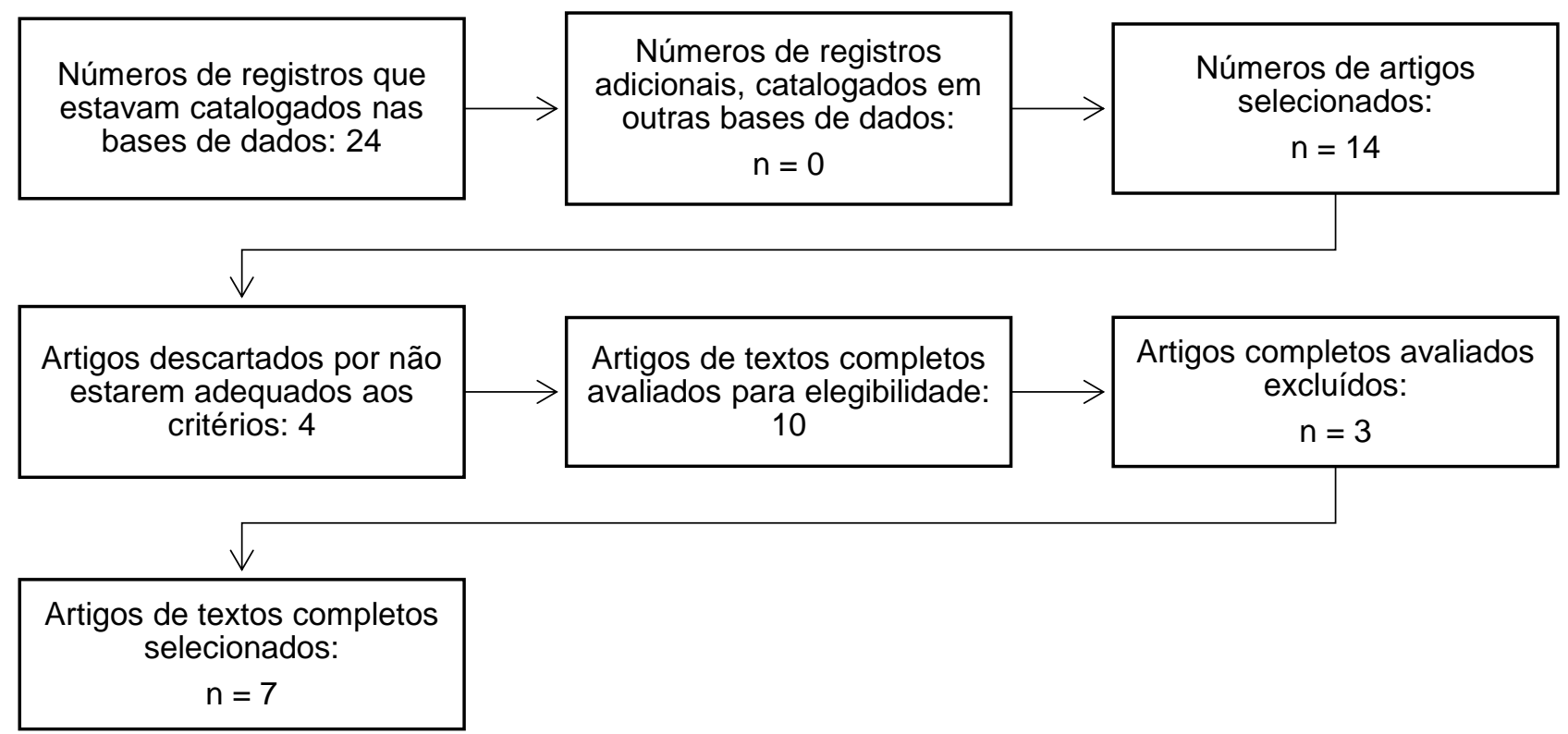

Fonte: Neto JMAS, et al., 2020.

No que se refere à população do Brasil, observa-se que as medidas de prestabilidade de serviços oferecidos pelo sistema único de saúde (SUS) está associada a determinados princípios onde procura fornecer serviços de saúde qualificado, humanitário, com tempo e acesso igualitário para todos os cidadãos. A rede de atenção à saúde é composta por estabelecer programas de atenção e serviços em um determinado conjunto de pessoas em determinada localização, tendo como critérios: atendimentos domiciliares dos quais estabelece os primeiros suportes para a pessoa que precisa, além de presta continuidade ao tratamento iniciado no hospital, as unidades de assistências básicas onde encontra-se múltiplos profissionais de áreas distintas, centro de especializações destinados a tratamentos específicos e os hospitais em casos mais graves. Essa abrangência possibilita mais conforto para a comunidade, uma vez que recebe e acolhe uma grande parcela dos habitantes, sobretudo as pessoas que possuem uma renda familiar inferior ao estabelecido dos quais não podem recorrer a uma assistência particular (BRASIL, 2002a).

Em nosso país, a alteração do antigo cenário referente a elaboração do planejamento público a médio e longo prazo tem ocasionado enorme adversidade referente aos aspectos sociais e econômicos, visto que o aumento da dimensão de pessoas que possuem a aptidão de usufruir mais anos de vida poucas vezes estão sendo seguida com uma boa qualidade de saúde. O avanço do envelhecimento da população desencadeou como decorrência, o grande aumento pela busca dos serviços públicos de saúde, além do acréscimo de distintas categorias do amparo no que se refere ao atendimento direcionado para pessoas com maiores idades vulneráveis ou oprimidos, como foi o caso das casas de apoio de longa estadia para os idosos. Trabalhos feitos ao longo do tempo descrevem que as casas dos quais os idosos se estabelecem na maioria das vezes se dar devido: a solidão por seu parceiro que já faleceu e não possuir expectativas de emprego dos quais é correlacionado a baixa renda dos mesmos, esses dois aspectos postos em pauta são os fatores mais relevantes nos últimos anos (CORACIN FL, 2012).

Ainda assim, o Brasil não possui medidas representativas relativas às atenções referentes a saúde bucal de idosos que vivem em casa de apoio, e muitos dos que vivem com a família não possuem um tratamento adequado, estando nos dias atuais bastante comum a falta de um protocolo padronizado referente aos procedimentos a serem realizados, existe carência no planejamento das atenções especializadas na odontogeriatria, como também a falta de informações muito das vezes pelos acompanhantes desses idosos. $\mathrm{Na}$ grande maioria dos casos, não dispõem de uma capacitação se quer, originando-se assim um cansaço mútuo entre o paciente e o cuidador, pois a uma precariedade de orientações e informações o suficiente para nortear a saúde bucal dos idosos. Encontram-se grande carência sobre os cuidados da cavidade oral tanto nas 
casas de apoio, quanto pelos próprios familiares, especialmente no que diz respeito às dificuldades mais comumente corriqueiras da mucosa oral, dado que muitos dos cuidadores observam a perda dos elementos dentais como algo normal quando se é correlacionado a velhice (MONTEIRO TAS, et al., 2013).

O elo referente à saúde deve ser integrado como um sistema de funcionamento onde atua de maneira correta, levando em consideração e prezando sempre pelo bem-estar físico, psicológico e de nível intelectivo do cidadão. Consequentemente, tanto a saúde bucal quanto a saúde geral correlacionam-se no aspecto de se uma não está boa prejudica de forma direta a outra.

Em circunstâncias em que o meio bucal não se encontra de forma adequada conseguirá estabelecer críticas adversidades diretamente na saúde geral, visto que influencia o aspecto nutricional referente à composição da alimentação adequada, as necessidades dos organismos em energias nutritivas e incapacidade da eficiência do aproveitamento biológico dos alimentos, prejudicando, desta maneira, o bem-estar como um todo, por consecutivo, minimiza a otimização de uma boa qualidade de vida e o contentamento da vida social do idoso. Com tal características, o estado geral referente à saúde do mesmo conseguirá acarretar na maioria das vezes patologias bucais. Com o transcorrer do tempo, existirá diminuição das coordenações motoras ocasionadas pelo avanço da idade, conseguindo prejudicar ainda mais o processo de higiene, do qual começa a ser feita de forma direta pelos familiares ou cuidadores (JANSSON MM, et al., 2017).

Nas literaturas a Odontologia geriátrica é citada como uma área onde abrange estabelecidas classes de entendimentos técnicos, desde pequenas a altas complicações, dos quais tem como objetivo melhorar os atendimentos com fins terapêuticos buscando sempre a melhor qualidade no tratamento e precauções de determinadas enfermidades. O cuidado com o paciente de terceira idade deve ser observado como um todo, não sendo analisada apenas a cavidade oral, muito menos sendo tratada de forma particular por cada especialidade, para a obtenção de um resultado benéfico e satisfatório para o paciente é necessário à interação e a participação de todos os membros que constituem o atendimento e a assistência, prezando sempre pela qualidade satisfatória de vida do paciente (MONTEIRO TAS, et al., 2013).

Por ser observada como uma área de especialização da odontologia dos quais é acompanhada há muito tempo, o CD possui domínio técnico e pratico na hora do atendimento, porém é de fundamental importância que sempre o profissional busque se atualizar, tendo uma visão não só de atendimentos clínicos e de unidades básicas de saúde, mas sim buscando atender também de forma satisfatória em hospitais, prestando os primeiros socorros porém é necessário que os mesmos demonstrem um bom desempenho dos quais essa área necessita (JANSSON MM, et al., 2017).

\section{Artigos localizados na base de dados}

Para o estabelecimento das ideias e das tabulações dos respectivos dados, foi estabelecido um instrumento de contribuição abrangendo: Autor, ano, objetivo, resultado e conclusão, dispondo de 07 artigos que atestaram os preceitos de inclusão, dos quais relatam a importância do Cirurgião Dentista no atendimento geriátrico e abaixo são elucidados pelo Quadro 1. 
Quadro 1 - Artigos relevantes para o desenvolvimento da pesquisa, com a finalidade de descrever a importância do Cirurgião Dentista ao atendimento aos pacientes idosos.

\begin{tabular}{|c|c|c|c|c|c|}
\hline Autor & Ano & Título & Objetivo & Resultado & Conclusão \\
\hline $\begin{array}{l}\text { MESTRINER } \\
\text { SF, et al. }\end{array}$ & 2014 & $\begin{array}{c}\text { Condições de } \\
\text { saúde bucal do } \\
\text { idoso no Brasil: } \\
\text { uma revisão de } \\
\text { literatura }\end{array}$ & $\begin{array}{l}\text { Este estudo teve como } \\
\text { objetivo analisar o estado de } \\
\text { saúde bucal e as condições } \\
\text { socioeconômicas dos idosos } \\
\text { usuários do Sistema Único de } \\
\text { Saúde (SUS) e sua relação } \\
\text { com um indicador subjetivo } \\
\text { dos impactos das condições } \\
\text { bucais na qualidade de vida. }\end{array}$ & $\begin{array}{l}\text { As frequências das dimensões do OHIP-14 } \\
\text { com maior impacto foram dor (13,16\%) e } \\
\text { sofrimento psicológico }(14,47 \%) \text {. A } \\
\text { associação de baixo impacto foi } \\
\text { representada por incapacidade social } \\
(71 \%) \text { e desvantagem social (63\%). }\end{array}$ & $\begin{array}{l}\text { Concluiu-se que os idosos que } \\
\text { relataram boa autopercepção de } \\
\text { saúde bucal e boa renda } \\
\text { expressaram a percepção de que a } \\
\text { saúde bucal reflete um bom impacto } \\
\text { na qualidade de vida. }\end{array}$ \\
\hline $\begin{array}{c}\text { MAGALHÃES } \\
\text { JHV, et al. }\end{array}$ & 2014 & $\begin{array}{l}\text { Caracterização da } \\
\text { pressão da língua } \\
\text { em idosos. }\end{array}$ & $\begin{array}{l}\text { Analisar a aplicação de } \\
\text { questionário, com registro da } \\
\text { presença ou ausência de } \\
\text { queixa de problemas na } \\
\text { deglutição, seguido da } \\
\text { avaliação miofuncional } \\
\text { orofacial, enfocando a } \\
\text { mobilidade e tensão de língua. }\end{array}$ & $\begin{array}{l}\text { Houve correlação moderada negativa entre } \\
\text { pico pressórico e idade. Os longevos } \\
\text { apresentaram a menor média de pico } \\
\text { pressórico e inferior ao padrão de } \\
\text { normalidade. Houve diferença entre a } \\
\text { média de pico pressórico e uso de } \\
\text { próteses, tensão de língua e mobilidade de } \\
\text { língua no estalo. }\end{array}$ & $\begin{array}{l}\text { Houve diminuição moderada do pico } \\
\text { pressórico da língua com o aumento } \\
\text { da idade e redução pressórica com o } \\
\text { decréscimo da tensão da língua e de } \\
\text { sua mobilidade no estalo. Em } \\
\text { contrapartida, os valores de pico } \\
\text { foram maiores nos idosos que } \\
\text { utilizam próteses dentárias, quando } \\
\text { comparados aos que destas não } \\
\text { fazem uso. }\end{array}$ \\
\hline $\begin{array}{l}\text { MORAIS EF, } \\
\text { et al. }\end{array}$ & 2014 & $\begin{array}{l}\text { Fatores } \\
\text { relacionados à } \\
\text { boca seca e } \\
\text { baixas taxas de } \\
\text { fluxo salivar em } \\
\text { idosos diabéticos: } \\
\text { uma revisão } \\
\text { sistemática da } \\
\text { literatura }\end{array}$ & $\begin{array}{l}\text { Realizar uma revisão } \\
\text { sistemática de estudos sobre } \\
\text { fatores relacionados à } \\
\text { xerostomia e / ou } \\
\text { hipossalivação em idosos com } \\
\text { diabetes. }\end{array}$ & $\begin{array}{l}\text { Alguns dos estudos encontraram relação } \\
\text { entre controle glicêmico deficiente e } \\
\text { hipossalivação. Outros descobriram que a } \\
\text { xerostomia era mais frequente em } \\
\text { pacientes que trabalhavam em ambientes } \\
\text { não domésticos e em } \\
\text { mulheres. Contudo, houve uma variação } \\
\text { considerável nos métodos utilizados pelos } \\
\text { pesquisadores nos estudos selecionados. }\end{array}$ & $\begin{array}{l}\text { Não conseguimos tirar conclusões } \\
\text { definitivas com base em nossa } \\
\text { análise dos estudos } \\
\text { selecionados. Estudos longitudinais } \\
\text { com tamanhos de amostra } \\
\text { adequados são necessários para } \\
\text { fornecer informações mais completas } \\
\text { sobre os fatores relacionados à } \\
\text { xerostomia e hipossalivação em } \\
\text { pacientes idosos com diabetes. }\end{array}$ \\
\hline
\end{tabular}

REAS/EJCH | Vol.Sup.n.51 | e3472 | DOI: https://doi.org/10.25248/reas.e3472.2020 Página 7 de 12 


\begin{tabular}{|c|c|c|c|c|c|}
\hline $\begin{array}{c}\text { COSTA DR, } \\
\text { et al. }\end{array}$ & 2015 & $\begin{array}{l}\text { Diadococinesia } \\
\text { oral e função } \\
\text { mastigatória em } \\
\text { idosos saudáveis. }\end{array}$ & $\begin{array}{c}\text { Relacionar os achados da } \\
\text { diadococinesia (DDC) oral } \\
\text { com a função mastigatória em } \\
\text { idosos saudáveis. }\end{array}$ & $\begin{array}{c}\text { A análise estatística demonstrou correlação } \\
\text { entre o tempo mastigatório e os parâmetros } \\
\text { coeficiente de variação do pico da } \\
\text { intensidade da sílaba "ta" e perturbações } \\
\text { do período da sílaba "ka", indicando } \\
\text { instabilidade da DDC. Para o tipo } \\
\text { mastigatório, apreensão e formação do } \\
\text { bolo alimentar não houve correlação com } \\
\text { os dados da DDC oral. }\end{array}$ & $\begin{array}{l}\text { A DDC oral correlacionou-se com o } \\
\text { tempo mastigatório em idosos } \\
\text { saudáveis, evidenciando que, quanto } \\
\text { maior a instabilidade na repetição } \\
\text { dos movimentos de ponta e dorso de } \\
\text { língua durante a produção } \\
\text { articulatória, maior o tempo } \\
\text { necessário para a preparação do } \\
\text { alimento para a deglutição. }\end{array}$ \\
\hline $\begin{array}{c}\text { MARTINS } \\
\text { AMEBL, et al. }\end{array}$ & 2015 & $\begin{array}{c}\text { Acesso a } \\
\text { informações } \\
\text { sobre como evitar } \\
\text { problemas de } \\
\text { saúde bucal entre } \\
\text { idosos registrados } \\
\text { na Estratégia de } \\
\text { Saúde da Família. }\end{array}$ & $\begin{array}{l}\text { Identificar a prevalência do } \\
\text { acesso a informações sobre } \\
\text { como evitar problemas bucais } \\
\text { entre idosos e verificar se esse } \\
\text { acesso foi maior entre os } \\
\text { residentes em domicílios } \\
\text { cadastrados na Estratégia de } \\
\text { Saúde da Família (ESF). }\end{array}$ & $\begin{array}{l}\text { Dentre os } 490 \text { participantes, } 53,4 \% \\
\text { relataram o acesso a informações sobre } \\
\text { como evitar problemas bucais. Esse } \\
\text { acesso foi maior entre os idosos que } \\
\text { residiam em domicílio cadastrado na ESF } \\
(2,04 / 1,14-3,67) \text {, tinham maior escolaridade } \\
(2,53 / 1,67-3,83) \text {, utilizaram os serviços } \\
\text { odontológicos por rotina ( } 1,84 / 1,07-3,17) \text {, } \\
\text { não relataram hábito tabagista atual ou } \\
\text { passado }(1,79 / 1,13-2,82) \text {, não possuíam } \\
\text { doença crônica }(2,14 / 1,34-3,42) \text { e não } \\
\text { tiveram impacto social das suas condições } \\
\text { de saúde bucal }(1,77 / 1,08-2,91) \text {. }\end{array}$ & $\begin{array}{c}\text { Conclui-se que a maioria dos idosos } \\
\text { teve acesso a informações sobre } \\
\text { como evitar problemas bucais, sendo } \\
\text { o acesso maior entre aqueles } \\
\text { cadastrados na Estratégia de Saúde } \\
\text { da Família. }\end{array}$ \\
\hline
\end{tabular}

REAS/EJCH | Vol.Sup.n.51 | e3472 | DOI: https://doi.org/10.25248/reas.e3472.2020 Página 8 de 12 


\begin{tabular}{|c|c|c|c|c|c|}
\hline RIGO L, et al. & 2015 & $\begin{array}{l}\text { Satisfação com a } \\
\text { vida, experiência } \\
\text { odontológica e } \\
\text { autopercepção da } \\
\text { saúde bucal entre } \\
\text { idosos. }\end{array}$ & $\begin{array}{c}\text { Analisar a relação existente } \\
\text { entre a satisfação com a vida } \\
\text { e a autopercepção em saúde } \\
\text { bucal e a experiência com } \\
\text { cirurgiões-dentistas. }\end{array}$ & $\begin{array}{l}\text { Os resultados mostraram que idosos com } \\
\text { maiores níveis de satisfação com a vida } \\
\text { apresentaram uma melhor percepção de } \\
\text { sua própria saúde bucal, bem como } \\
\text { possuem uma melhor imagem percebida } \\
\text { dos cirurgiões-dentistas e sentem menos } \\
\text { ansiedade em relação à experiência com o } \\
\text { dentista. }\end{array}$ & $\begin{array}{c}\text { Comprova-se que tanto a } \\
\text { autopercepção que os idosos } \\
\text { possuem da saúde bucal quanto a } \\
\text { experiência com cirurgiões-dentistas } \\
\text { possuem uma associação com a } \\
\text { satisfação com a vida. }\end{array}$ \\
\hline $\begin{array}{c}\text { SILVA BLA, } \\
\text { et al. }\end{array}$ & 2015 & $\begin{array}{l}\text { Condição de } \\
\text { saúde bucal de } \\
\text { idosos } \\
\text { institucionalizados } \\
\text { em } \\
\text { Araguaína/TO. }\end{array}$ & $\begin{array}{l}\text { Relatar as condições de saúde } \\
\text { bucal do idoso brasileiro, } \\
\text { enfatizando a qualidade de } \\
\text { vida desse idoso, bem como o } \\
\text { conhecimento dos cuidadores } \\
\text { no que diz respeito à higiene e } \\
\text { manutenção da saúde bucal } \\
\text { dessa população. }\end{array}$ & $\begin{array}{l}\text { Ao realizar a triagem desses artigos, } \\
\text { utilizando-se os critérios de inclusão, têm- } \\
\text { se para o estudo um total de doze artigos } \\
\text { trabalhados, sendo sete }(58,3 \%) \text { dos } \\
\text { artigos publicados em } 2014 \text { e cinco }(41,7 \%) \\
\text { dos artigos publicados em } 2015 \text {. }\end{array}$ & $\begin{array}{l}\text { Pode-se concluir com esse trabalho } \\
\text { que existe a necessidade de } \\
\text { tratamento odontológico voltado para } \\
\text { essa população, devido às } \\
\text { patologias oriundas do próprio } \\
\text { envelhecimento. }\end{array}$ \\
\hline
\end{tabular}

Fonte: Neto JMAS, et al., 2020. 
A prioridade como um bem pertencente a todos principalmente no que se refere à saúde oral é de suprema relevância para o contexto da saúde como um todo em indivíduos idosos, podendo ser constado que dessa forma a parte física, psicológica e social do paciente ficam em harmonia, tornando-os na maioria das vezes mais proativos (RIGO L, et al., 2015). Encontrar-se nos dias atuais uma diminuição da falta de compreensão ao que se refere aos imprescindíveis tratamento terapêuticos na área da odontologia, na maioria das vezes pelos próprios pacientes idosos, em tal grau encontra-se também pelos próprios familiares ou cuidadores nas casas de repouso, além das pessoas que trabalham no apoio e por fim porém não menos importante os profissionais que consultam e cuidam deles. Os pacientes geriátricos devem ser conscientizados é através disso é de suma importância a sua conscientização para manter os cuidados bucais de forma continua, mesmo aqueles paciente que apresentem poucos dentes ou até mesmo nenhum dente, deixando claro para eles que a higienização da cavidade oral não se restringe apenas aos dentes, mas sim a todas as estruturas que formam a cavidade oral.

É crucial a transparência de abordagem, a forma de visão, de compreensão que o profissional deve ter ao falar com eles, os idosos possuem a necessidade se sentir confortável e o Cirurgião Dentista deve conseguir conquistar a confiança deles (SILVA BLA, et al., 2015).

Do mesmo modo, é bastante considerável que no âmbito familiar em que o idoso resida todos os membros estejam atentos seja qual for às alterações correspondentes aos hábitos ou até mesmo o comportamento do idoso, levando como principais critérios na hora de analisar: possíveis modificações na fala, a forma de se expressar, possuir expressões que levem a entender que os mesmos estão com alguma dor, a falta de apetite, esses aspectos relacionados a saúde do paciente geriátrico deve ser cruciais e ter que sempre ser analisado, isso ocorre porque o paciente especifico dessa modalidade pode não observar possíveis alterações que estão acontecendo com eles, e existem os que sabem que possuem alguma coisa de anormal, porém não estabelece uma devida importância ou protelam o problema, tornando-se assim um fator determinante quando os mesmos possuem alguma enfermidade (SILVA BLA, et al., 2015).

Uma vez que o paciente de terceira idade chega ao consultório odontológico, o CD não deve tratar como pessoas frágeis, debilitadas, mas sim como um paciente normal que possui suas particularidades e complexidades, com as conjunturas físicas demonstradas por conta dos efeitos e mudanças que conduzem ao envelhecimento. Referente aos princípios mentais desses pacientes, geralmente os pacientes que vão ao consultório odontológico são indivíduos que apresenta maior quantidade de conhecimento dos quais foram estabelecidas ao longo dos anos vividos, sua forma sociável e comunicativa, irá necessitar a princípio dos primeiros contatos adquiro entre o profissional e o paciente, porém dois fatores são levados em consideração: o bem-estar físico e psicológico do paciente. Estudos demonstram que a boa interação com o idoso é de suma importância para que se obtenha do mesmo as informações desejadas, assegurando-lhe segurança e elevando o contentamento no que concerne a assistência e o suporte a saúde deles (COSTA DR, et al., 2015).

A situação no que se refere a saúde geral do idoso necessitam serem analisadas com aspectos detalhados para que inexistam enfermidades e modificação do quadro que possam vim a intervir nos recursos terapêuticos. É recomendado em todo tempo atentar a necessidade de conduzir ao profissional de saúde para que esse assegure as situações de saúde do paciente e comprove se o mesmo está hábil ou não para a consumação do tratamento proposto pelo CD.

Um relatório recente deve ser feito no que se refere a um programa de prevenção, buscando dessa maneira evitar contratempos futuros ou, no mínimo, reduzindo a probabilidade de interferências possam influenciar ainda mais a saúde do idoso (RIGO L, et al., 2015).

O CD tem que buscar uma forma de atendimento correto e preciso, buscando dar todo o suporte, dedicação e conhecimento tanto para os cuidadores, ou responsáveis quanto para o paciente se for necessário. Todavia, eles devem ser conhecimento que precisam fazer sua parte, visto que não obtém um resultado satisfatório se não for estabelecido de forma benéfica a aceitação do tratamento e ajuda para o êxito do tratamento proposto. As atividades de profissionais especializados em clinicas especializadas para o atendimento desses pacientes, desde unidades públicas como particulares, dos quais podem fornecer um serviço integral de saúde bucal, como é o caso dos procedimentos odontológicos, buscando, desta forma, a um resultado auspicioso (MARTINS 
AMEBL, et al., 2015). É bastante relevante demonstrar a importância e a necessidade de um odontogeriatra no atendimento, além de humanizado, prestativo e atencioso, tanto no atendimento em clinicas como na integração de atendimentos hospitalares, buscando sempre trabalhar com equipe multiprofissional e até mesmo estabelecendo e fortalecendo programas de âmbito regenciais que busquem estabelecer a saúde bucal dessas pessoas (MAGALHÃES JHV, et al., 2014).

No que se refere à importância do acompanhamento do Cirurgião Dentista ao paciente idoso, observa-se que os cuidados multiprofissionais possuem uma construção bastante significativa ao sucesso dos tratamentos, no entanto à uma imposição ao que se refere a algumas reformulações de visão antiga dos quais devem serem levadas em consideração, para só assim os modernos sistemas serem realizados, do qual considera-se como de fundamental importância a assiduidade da odontogeriatria (MORAIS EF, et al., 2014). Tem que se perceber que a associação do entendimento das características especificas de cada profissional é parte de uma razão decisiva para a benfeitoria e especialização do conhecimento técnico, para deste modo atingir um prognostico minucioso e obter diagnósticos precisos, objetivando em todo tempo a atenuação de tempo, qualidade de vida do idoso e melhoria respectivamente, como pode ser observado na Figura 1 (MESTRINER SF, et al., 2014).

Figura 1 - Apresentação do fenômeno da reflexão da importância do cirurgião-dentista nos cuidados geriátricos através de uma formação interdisciplinar (MESTRINER SF, et al., 2014).

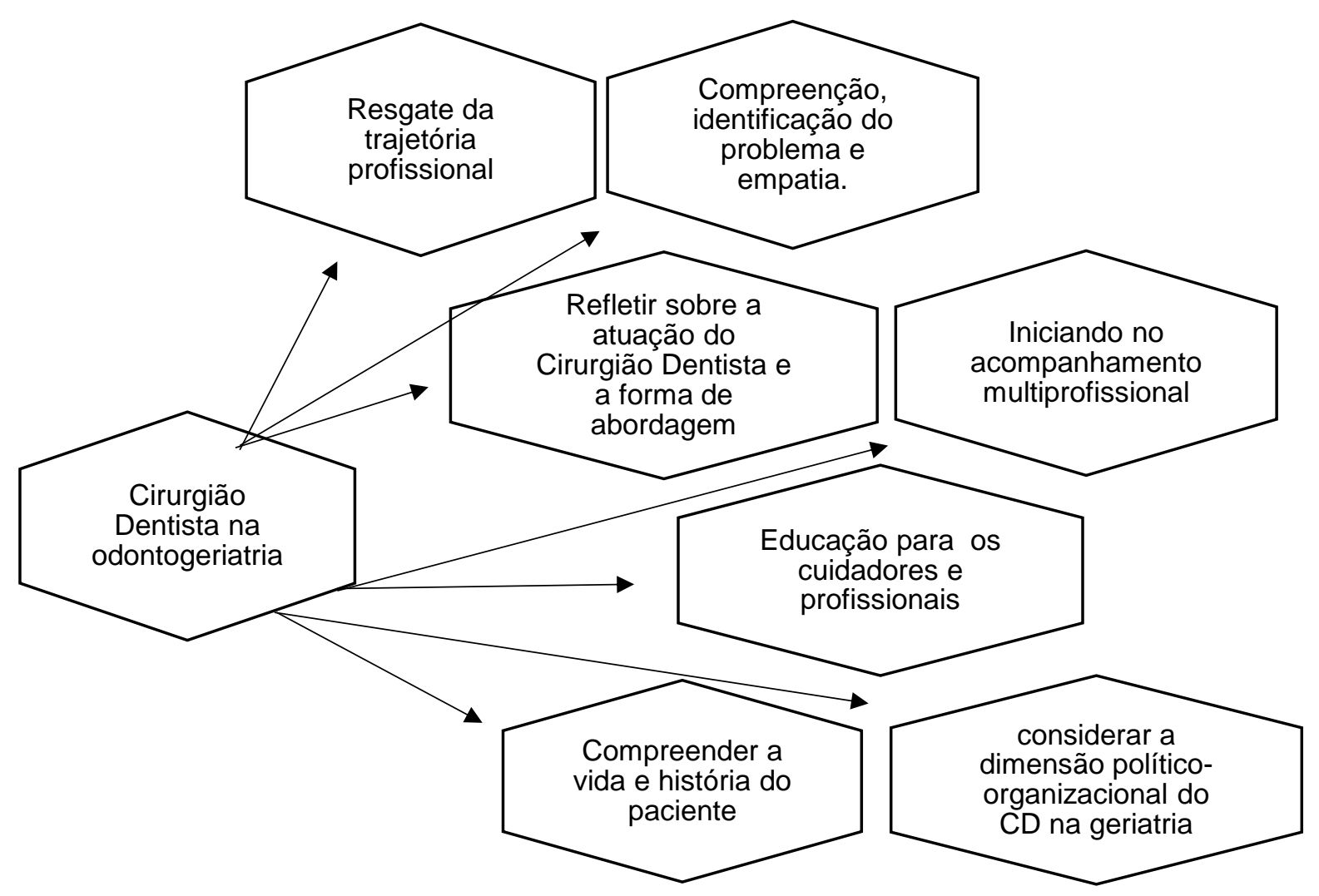

Fonte: Neto JMAS, et al., 2020. Baseado de Mestriner SF, et al., 2014.

\section{CONSIDERAÇÕES FINAIS}

Fica notório a imprescindível atuação multiprofissional, além do acompanhamento dos familiares ou cuidadores, no que se expõe aos manejos dos tratamentos dos pacientes geriátricos. Procedimentos característicos para essa faixa etária devem ser estabelecidos no aspecto de aperfeiçoar ainda mais o atendimento e a continuidade de todo tratamento, visto que no Brasil a crescente população de pessoas idosas tem aumentado de forma vertiginosa e o cirurgião dentista deve situar-se a essas circunstâncias e amplificar suas compreensões na área da odontogeriatria para só assim assegurar um recurso terapêutico preciso, buscando exatidão nos prognósticos e diagnósticos. 


\section{REFERÊNCIAS}

1. AGOSTINHO ACMG, et al. Edentulismo, uso de prótese e autopercepção de saúde bucal entre idosos. Rev Odontol UNESP. 2015; 44(2): 74-79.

2. AMES NJ, et al. Effects of Systematic Oral Care in Critically III Patients: A Multicenter Study. Am J Crit Care. 2011; 20(5): 2011: 103-14.

3. BARBOSA LNF, et al. Neuropsicologia e perspectivas para o cuidado integral de idosos. Saúde do Idoso: uma abordagem multidisciplinar, 2015; 215-234.

4. BERKEY DB e SCANNAPIECO FA. Medical considerations relating to the oral health of older adults. Spec Care Dentist, Malden. 2013; 33(4): 164-176.

5. BIZERRIL DO, et al. Papel do cirurgião-dentista nas visitas domiciliares:atenção em saúde bucal. Rev Bras Med Fam Comunidade. 2016; 10(37): 1-8.

6. BRASIL. Ministério da Educação. CNE. Conselho Nacional de Educação. Câmara de Educação Superior. Resolução CNE-CES 3, de 19/02/2002 Institui as Diretrizes Curriculares Nacionais do Curso de Graduação em Odontologia. Diário Oficial da União, Brasília 04 de março de 2002. Seção 1, p. 10.

7. CORACIN FL. Interdisciplinaridade. Medicina Bucal: a prática da Odontologia Hospitalar. São Paulo: Santos, 2012; 2527: $315 p$.

8. COSTA DR, et al. Diadococinesia oral e função mastigatória em idosos saudáveis. Audiol Commun Res. 2015; 20(3): 191-7.

9. JANSSON MM, et al. Effects of simulation education on oral care practices - a randomized controlled trial. Nurs Crit Care. 2017; 22 (3): 161-8.

10. MAGALHÃES JHV, et al. Caracterização da pressão da língua em idosos. Audiol Commun Res. 19(4), 2014 ; $375-9$.

11. MARTINS AMEBL, et al. Access to information on how to avoid oral health problems among elderly persons registered with the Family Health Strategy. Rev Bras Geriatr Gerontol. 2015; 18(4): 855-9.

12. MENDES E. As redes de atenção à saúde. Brasília: Organização Pan-Americana de Saúde, 2011.

13. MESTRINER SF, et al. Condições de saúde bucal e qualidade de vida de idosos usuários do Sistema Único de Saúde. RGO. 2014; 62(4): 389-94.

14. MONTEIRO TAS, et al. Cuidadores de idosos e atitudes frente à saúde bucal: uma revisão integrativa. Rev Enferm UFPI. 2(spe): 2013; $102-7$.

15. MORAES E. Atenção à saúde do Idoso: aspectos conceituais. Brasília: Organização Pan-Americana da Saúde, 2012.

16. MORAIS EF, et al. Factors related to dry mouth and low salivary flow rates in diabetic elderly: a systematic literature review. Rev Bras Geriatr Gerontol. 2014; 17(2): 417-23.

17. RABELO GD, et al. Atendimento odontológico ao paciente em unidade de terapia intensiva. Arq Med Hosp Cienc Med Santa Casa São Paulo. 2010; 55(2): 67-70.

18. RIGO L, et al. Satisfação com a vida, experiência odontológica e autopercepção da saúde bucal entre idosos. Ciênc saúde coletiva. 2015; 20(12): 3681-8.

19. SILVA BLA, et al. Condição de saúde bucal de idosos institucionalizados em Araguaína/TO. Braz J Periodontol. 2015; 25(1): 7-13.

20. SOWMYA MK, et al. Psychological impact of edentulousness. JIADS. 2011; 2(1), 34-36. 